\title{
EphB Maintains Dendritic Spine Morphology through Focal Adhesion Kinase
}

\author{
Yu Chen* and Wing-Yu Fu* \\ Department of Biochemistry, Molecular Neuroscience Center and Biotechnology Research Institute, Hong Kong University of Science and Technology, \\ Clear Water Bay, Hong Kong, China \\ Review of Shi et al.
}

The postsynaptic components of excitatory synapses are located at specialized structures called dendritic spines, small protrusions along neuronal dendrites. Dendritic spines are not static, and changes in spine size, shape, and density are believed to be associated with synaptic plasticity and memory. A plethora of molecules have been found to regulate dendritic spine morphogenesis, including cytoplasmic kinases, scaffold proteins, cell surface receptors, and ion channels.

Eph receptors, including EphAs and EphBs, form the largest family of receptor tyrosine kinases. Their membrane-attached ligands ephrins, not only activate Eph receptors on the opposing cell membrane and transduce forward signaling, but also elicit reverse signaling cascades in ephrinexpressing cells upon binding to the receptors. Ephs and ephrins have been well studied in axon pathfinding and topographic mapping. In recent years, accumulating evidence demonstrate that Eph receptors play important roles in the mor-

Received Aug. 24, 2009; revised Sept. 8, 2009; accepted Sept. 9, 2009

Y.C. and W.-Y.F. are postdoctoral fellows in the laboratory of Nancy Y. Ip, whose studies were supported in part by the Research Grants Council of Hong Kong (HKUST1/06C, 6421/05M, 6444/06M, 6431/06M, and 661007), the Area of Excellence Scheme of the University Grants Committee (AoE/ B-15/01), and the Hong Kong Jockey club. We thank Ka-Chun Lok for his help on preparing the figures, and Prof. Nancy Y. Ip and Drs. Amy K. Y. Fu and Kwok-On Lai for their critical reading of the manuscript.

${ }^{*} Y$.C. and W.-Y.F. contributed equally to this work.

Correspondence should be addressed to either Y.Chen or W.-Y. Fu at the above address, E-mail: bcyuchen@ust.hk or bowyfu@ust.hk.

D01:10.1523/JNEUROSCI.4155-09.2009

Copyright $\odot 2009$ Society for Neuroscience $\quad$ 0270-6474/09/2913091-03\$15.00/0 phogenesis of dendritic spines (Pasquale, 2005; Chen et al., 2009). For example, activation of EphB signaling induces the formation of dendritic spines. In contrast, EphA4 triggers dendritic spine retraction in mature neurons, and knock-out of EphA4 in hippocampal neurons results in aberrant dendritic spine morphology.

Although the mechanisms by which EphB receptors regulate spine formation have been extensively studied, it is not clear whether EphB receptors play a role in the maintenance of dendritic spines and how they exert their actions. An early study on EphB1-3 triple-knock-out mice revealed that the loss of EphB receptors reduces spine number in mature neurons, suggesting that EphBs either promote spine formation or are required for maintaining mature dendritic spines (Henkemeyer et al., 2003). It was recently demonstrated that knockdown of EphB2 by shRNA during the third week in vitro causes a shift of spine morphology from mushroom-shaped to long filopodia-like protrusions, supporting a role of EphB2 in stabilizing mature dendritic spines (Kayser et al., 2008). Thus, it would be of interest to elucidate how EphB receptors maintain the shape of mature dendritic spines.

In a recent issue of The Journal of Neuroscience, Shi et al. (2009) addressed this question by demonstrating that EphB2 maintains the morphology of mature dendritic spines by suppressing the actindestabilizing activity of cofilin through focal adhesion kinase (FAK) and the small
GTPase RhoA. Previous work from the same group showed that FAK-dependent EphB signaling is involved in dendritic filopodia shortening and spine formation/ maturation in young neurons (Moeller et al., 2006). The authors now report that a similar EphB-FAK-RhoA pathway functions in mature neurons, and they delineate the signaling events downstream of RhoA in the maintenance of mature dendritic spines.

Inhibition of EphB-FAK-RhoA signaling in mature neurons results in the reversal of mushroom-shaped spines to filopodia-like structures. Although an increase of filopodia-like protrusions are seldom observed in mature neurons except in specific conditions such as neuronal injury, these protrusions are frequently found in young neurons, which are highly mobile in search of presynaptic partners. Thus, it is interesting to speculate that EphB-FAKRhoA signaling in mature neurons may contribute to synaptic plasticity in adulthood.

Shi et al. (2009) used a Cre-loxP recombination system to knock out FAK in neurons, and provided evidence that loss of FAK function induces a shift of mature dendritic spines to long filopodia-like protrusions. Interestingly, the authors showed that some filopodia-like spines in $\mathrm{fak}^{-1-}$ mature neurons receive multiple inputs from presynaptic terminals, as revealed by synaptophysin staining. Similarly, multiple PSD-95 puncta were found in some spines, suggesting that synapse formation/maturation is affected in $f a k^{-1-}$ neurons [Shi et al. (2009), their Fig. 2 and 
supplemental Fig. 3]. As the innervation of postsynaptic neuron by presynaptic terminals is critical for synaptic transmission and plasticity, it would be interesting to examine whether presynaptic and postsynaptic apparatus are properly aligned in $\mathrm{fak}^{-l-}$ neuron by costaining with antibodies against presynaptic and postsynaptic markers. It is also important to investigate whether synaptic transmission is abnormal in the knock-out neurons by measuring miniature EPSCs.

Because EphB2 was shown to regulate FAK, Shi et al. (2009) hypothesized that EphB2 acts upstream of FAK in maintaining mature dendritic spines. Blockade of EphB receptor-dependent signaling by expressing an EphB2 kinase-inactive mutant (dnEphB2) resulted in the formation of many filopodia-like protrusions instead of mushroom-shaped spines [Shi et al. (2009), their Fig. 6A,B]. Because dnEphB2 has an intact and functional extracellular domain that binds ephrins at presynaptic terminals, the defective phenotype could be caused either by the direct blockade of EphB forward signaling at postsynaptic sites or by activating ephrin-B reverse signaling at presynaptic neurons. A similar phenotype is observed in EphB2 knockdown neurons, in which both forward and reverse signaling are disrupted (Kayser et al., 2008), thus suggesting that the defective spine phenotype is attributable to EphB forward signaling. Interestingly, the abnormal dendritic spine morphology in dnEphB2-expressing neurons can be rescued by expression of a constitutively active mutant form of FAK [Shi et al. (2009), their Fig. $6 C$ ], supporting a role for FAK as a downstream regulator of EphB2 in maintaining mature dendritic spines. On the other hand, the multiple innervation and branched spine morphology in $\mathrm{fak}^{-1-}$ neurons is not observed in neurons expressing dnEphB2 [Shi et al. (2009), their Fig. 4, Fig. 6]. Therefore, in addition to the pathway described by Shi et al. (2009), FAK may also regulate spine and synapse development through a mechanism that is independent of EphB2.

The next question addressed by Shi et al. (2009) was how FAK regulates EphB2dependent spine morphology. FAK constitutively associates with integrins in neurons, although the functional roles of this association in dendritic spine morphogenesis are not clear. Shi et al. (2009) found that ephrin-B2 stimulates the binding of FAK to EphB2 and reduces the interaction between FAK and integrin, which may result in an inhibition of integrin signaling in mature neurons [Shi

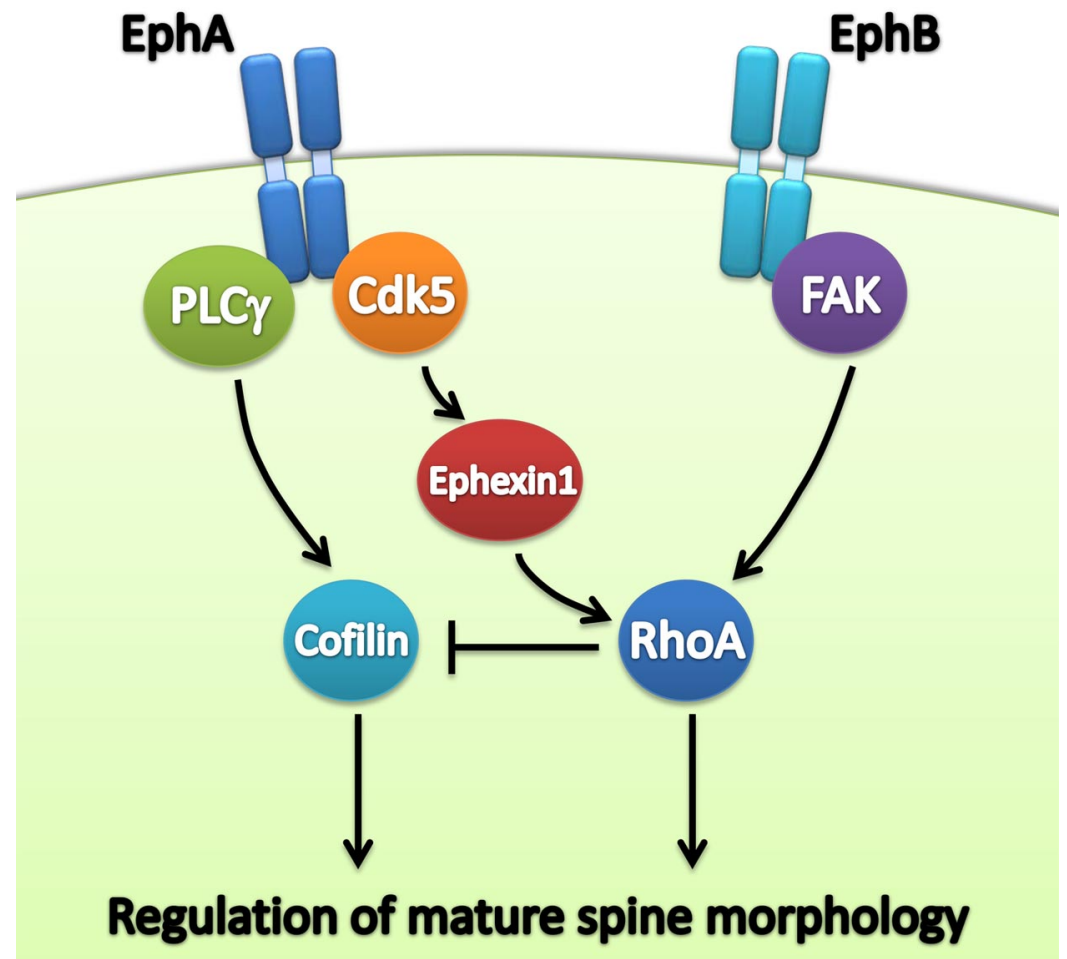

Figure 1. Regulation of dendritic spine morphology by EphA and EphB receptors in mature neurons. EphA receptors enhance cofilin activity through $\mathrm{PLC} \gamma$, leading to the retraction of dendritic spines. Alternatively, EphA receptors induce RhoA activation and spine retraction through a mechanism dependent on C $d k 5$ (cyclin-dependent kinase 5) and a guanine nucleotide exchange factor ephexin1. On the other hand, EphB receptors inactivate cofilin through FAK-dependent activation of RhoA, which stabilizes actin cytoskeleton and maintains the morphology of mature spines.

et al. (2009), their Fig. 5]. This finding is consistent with a previous report that activation of integrin signaling induces elongation of dendritic spines and formation of new filopodia in hippocampal neurons (Shi and Ethell, 2006). However, direct evidence for the roles of integrin signaling in EphB-FAK-mediated maintenance of mature spines awaits further study.

What are the signaling events that occur downstream of FAK to maintain mature spines? By expressing mutated forms of FAK that cannot interact with regulators of Rho GTPases in $\mathrm{fak}^{-/-}$neurons, Shi et al. (2009) demonstrated that Rho GTPases are required for FAK-dependent spine maintenance [Shi et al. (2009), their Fig. 4]. RhoA, which acts downstream of FAK, has been shown to stabilize actin cytoskeleton through LIMK-1-dependent inactivation of cofilin, an actin-depolymerizing factor. Therefore, Shi et al. (2009) further showed that cofilin inactivation was indispensable in EphB-FAK-mediated spine maintenance [Shi et al. (2009), their Fig. 7, Fig. 8, Fig. 10].

It is interesting to note that multiple signaling molecules that function in EphB-dependent spine maintenance also play critical roles in EphA-mediated signaling. For example, EphA receptors have been shown to regulate FAK signaling (Pasquale, 2005), underscoring the importance of investigating whether FAK is also a target of EphA in mature neurons and whether FAK serves as a coordinator to control the EphA-mediated spine retraction and EphB-dependent spine maintenance. Furthermore, while inhibition of integrin signaling by EphA4 was found to be essential for ephrin-A-induced spine retraction (Bourgin et al., 2007), Shi et al. (2009) showed that activation of EphB might also lead to suppression of integrin signaling. Possible explanations for this discrepancy are that EphB and EphA receptors regulate integrin signaling through recruitment of different downstream signaling molecules, or EphB2 triggers other signaling pathways that could compensate for the effect of integrin inhibition in mature spines. Interestingly, EphA activation facilitates the release of cofilin from the membrane to a cytosolic pool, leading to activation of cofilin and actin depolymerization, which contributes to EphAdependent spine retraction (Zhou et al., 2007). This finding, together with the vital role of cofilin inactivation in EphB- 
dependent spine maintenance suggests that cofilin is an integrator of EphA and EphB signaling in modulating mature spine morphology. However, RhoA, the Rho GTPase that reduces cofilin activity to maintain mature spines, can also trigger actin depolymerization and spine retraction through the regulation of actomyosin contractibility. A previous study of EphA4 signaling suggests that instead of maintaining spines, RhoA activation is essential for EphA4-dependent dendritic spine retraction (Fu et al., 2007), raising a question of how RhoA plays its role in spine maintenance. Investigating whether EphA and EphB receptors activate different pools of RhoA and how they trigger RhoA downstream signaling differentially in mature neurons would be helpful to resolve the above discrepancy. Moreover, it is interesting to speculate that spine morphology can be regulated dynamically by fine-tuning the activity of critical signaling molecules through the coordinated activation of EphA and EphB receptors (Fig. 1).

Another interesting finding by Shi et al. (2009) is the increased dendritic spine motility in $\mathrm{fak}^{-1-}$ neurons [Shi et al. (2009), their supplemental Fig. 4]. The motility of dendritic spines is associated with NMDA receptor-dependent synaptic plasticity (Alvarez et al., 2007). Pharmacological inhibition or knockdown of NMDA receptor NR1 subunit enhances spine motility, which is also observed in $\mathrm{fak}^{-1-}$ neurons. As the regulator for both NMDA receptor and FAK, EphB2 promotes filopodia motility in young neurons (Kayser et al., 2008), while its role in regulating dendritic spine motility in mature neurons has not been investigated. Thus it would be of interest to examine whether and how EphB2 coordinates with NMDA receptor and FAK to regulate spine motility.

In summary, the findings by Shi et al. (2009) reveal a novel pathway in maintaining the morphology of mature dendritic spines. Dendritic spine morphogenesis is associated with long-term changes of synaptic plasticity. Thus it can be envisaged that interfering EphB-FAK signaling in mature dendritic spines would affect synaptic functions. Future studies on the regulation of synaptic protein composition and synaptic plasticity by EphB-FAK pathway would definitely provide further insights into molecular basis of learning and memory.

\section{References}

Alvarez VA, Ridenour DA, Sabatini BL (2007) Distinct structural and ionotropic roles of NMDA receptors in controlling spine and synapse stability. J Neurosci 27:7365-7376.

Bourgin C, Murai KK, Richter M, Pasquale EB (2007) The EphA4 receptor regulates dendritic spine remodeling by affecting beta1integrin signaling pathways. J Cell Biol 178: 1295-1307.

Chen Y, Fu AK, Ip NY (2009) Bidirectional sig- naling of ErbB and Eph receptors at synapses. Neuron Glia Biol, in press.

Fu WY, Chen Y, Sahin M, Zhao XS, Shi L, Bikoff JB, Lai KO, Yung WH, Fu AK, Greenberg ME, Ip NY (2007) Cdk5 regulates EphA4-mediated dendritic spine retraction through an ephexin1dependent mechanism. Nat Neurosci 10:67-76.

Henkemeyer M, Itkis OS, Ngo M, Hickmott PW, Ethell IM (2003) Multiple EphB receptor tyrosine kinases shape dendritic spines in the hippocampus. J Cell Biol 163:1313-1326.

Kayser MS, Nolt MJ, Dalva MB (2008) EphB receptors couple dendritic filopodia motility to synapse formation. Neuron 59:56-69.

Moeller ML, Shi Y, Reichardt LF, Ethell IM (2006) EphB receptors regulate dendritic spine morphogenesis through the recruitment/phosphorylation of focal adhesion kinase and RhoA activation. J Biol Chem 281:1587-1598.

Pasquale EB (2005) Eph receptor signalling casts a wide net on cell behaviour. Nat Rev Mol Cell Biol 6:462-475.

Shi Y, Ethell IM (2006) Integrins control dendritic spine plasticity in hippocampal neurons through NMDA receptor and $\mathrm{Ca}^{2+} /$ calmodulindependent protein kinase II-mediated actin reorganization. J Neurosci 26:1813-1822.

Shi Y, Pontrello CG, DeFea KA, Reichardt LF, Ethell IM (2009) Focal adhesion kinase acts downstream of EphB receptors to maintain mature dendritic spines by regulating cofilin activity. J Neurosci 29:8129-8142.

Zhou L, Martinez SJ, Haber M, Jones EV, Bouvier D, Doucet G, Corera AT, Fon EA, Zisch AH, Murai KK (2007) EphA4 signaling regulates phospholipase $\mathrm{C} \gamma 1$ activation, cofilin membrane association, and dendritic spine morphology. J Neurosci 27:5127-5138. 